\title{
Ingestion of Chlorella Reduced the Oxidation of Erythrocyte Membrane Lipids in Senior Japanese Subjects
}

\author{
Taiki Miyazawa ${ }^{1}$, Kiyotaka Nakagawa $^{1}$, Hideo Takekoshi ${ }^{2}$, Ohki Higuchi ${ }^{3}$, Shunji Kato ${ }^{1}$, \\ Momoko Kondo ${ }^{1}$, Fumiko Kimura ${ }^{1}$ and Teruo Miyazawa ${ }^{1 *}$ \\ ${ }_{1}^{1}$ Food and Biodynamic Chemistry Laboratory, Graduate School of Agricultural Science, Tohoku University (Sendai 981-8555, Japan) \\ ${ }^{2}$ Production \& Development Department, Sun Chlorella Co., Ltd. (Kyoto 600-8177, Japan) \\ ${ }^{3}$ Biodynamic Plant Institute Co., Ltd. (Sapporo 001-0021, Japan)
}

\begin{abstract}
Accumulation of phospholipid hydroperoxide (PLOOH) in erythrocyte membranes is an abnormality found in patients with senile dementia, including those with Alzheimer's disease. In our previous studies, dietary xanthophylls (polar carotenoids such as lutein) were hypothesized to inhibit lipid peroxidation. In the present study, we conducted a randomized, double-blind, placebo-controlled human trial to assess the impact for a total of 2 months Chlorella supplementation (8 $\mathrm{g}$ Chlorella/day/person; equivalent to $22.9 \mathrm{mg}$ lutein/day/person) on PLOOH and carotenoid concentrations in erythrocytes as well as plasma of 12 normal senior subjects. After 1 or 2 months of treatment, erythrocytes and plasma lutein concentrations increased in the Chlorella group but not in the placebo group. In the Chlorella-supplemented group, erythrocyte PLOOH concentrations after a total of 2 months of treatment were lower than the concentrations before supplementation. These results suggest that Chlorella ingestion improved erythrocyte antioxidant status and lowered PLOOH concentrations. These reductions might contribute to maintaining the normal function of erythrocytes and prevent the development of senile dementia.
\end{abstract}

Key words: Chlorella, antioxidant, carotenoids, lipid oxidation, erythrocytes, senile dementia

\section{INTRODUCTION}

Senile dementia, including Alzheimer's disease (AD), is a serious neurodegenerative disorder in humans ${ }^{1)}$. Erythrocytes of $\mathrm{AD}$ patients are known to be in an excessively oxidized state ${ }^{2,3)}$. In 1992, Miyazawa et al. ${ }^{4)}$ investigated phospholipid hydroperoxide (PLOOH) concentrations as a specific marker for membrane lipid oxidative injury and discovered that erythrocyte in $\mathrm{AD}$ patients were significantly higher than in normal control subjects. Erythrocytes having high levels of PLOOH have been recognized as aged cells. It has been suggested that such cells might have a decreased ability to transport oxygen to the brain and could also impair blood rheology, thus facilitating senile dementia $^{5-7)}$. We considered that oxygen dissociation from oxidized hemoglobin may be inhibited by the presence of $\mathrm{PLOOH}$ in the phospholipid bilayers in the PLOOH-rich erythrocytes $^{5-7)}$.

For human erythrocytes, $\alpha$-tocopherol and the xanthophylls (polar carotenoids such as lutein) are important lipo- philic antioxidants ${ }^{8}$. In our recent studies ${ }^{9)}$, erythrocyte lutein concentrations in $\mathrm{AD}$ patients were found to be significantly lower than in control subjects, and an inverse relationship was confirmed between erythrocyte lutein and $\mathrm{PLOOH}$ concentrations in the $\mathrm{AD}$ patients. In our murine study $^{10)}$, oral administration of a lutein concentrate showed its incorporation into erythrocytes and distribution in the phospholipid bilayers. This treatment resulted in decreased erythrocyte PLOOH levels. We also confirmed in a human study that oral intake of lutein capsules increased lutein concentrations and prevented PLOOH accumulation in erythrocytes ${ }^{11}$. On the basis of our data obtained in human and animal studies, we suggested that dietary xanthophyll like lutein has the potential to act as an important antioxidant in erythrocytes. We further hypothesized that it could contribute to the suppression of PLOOH accumulation in erythrocyte membranes and might therefore reduce $\mathrm{AD}$. Thus, in daily life, continuous dietary intake of foods rich in lutein might help prevent the development of $\mathrm{AD}$.

\footnotetext{
*Correspondence to: Teruo Miyazawa Ph.D., Food and Biodynamic Chemistry Laboratory, Graduate School of Agricultural Science, Tohoku University, Sendai 981-8555, JAPAN

E-mail: miyazawa@ biochem.tohoku.ac.jp

Accepted May 10, 2013 (received for review May 7, 2013)

Journal of Oleo Science ISSN 1345-8957 print / ISSN 1347-3352 online

http://www.jstage.jst.go.jp/browse/jos/ http://mc.manusriptcentral.com/jjocs
} 


\section{T. Miyazawa, K. Nakagawa, H. Takekoshi et al.}

However, to the best of our knowledge, no extensive study of food resources that can efficiently prevent erythrocyte lipid oxidation has been undertaken.

Chlorella pyrenoidosa is a unicellular green alga ${ }^{12)}$ rich in protein, minerals and lutein ${ }^{13-15)}$. In the present study, we hypothesized that continuous dietary intake of Chlorella might prevent PLOOH accumulation in the erythrocytes of senior Japanese subjects. To evaluate the hypothesis, we performed a randomized, double-blind, placebo-controlled human trial to investigate the efficacy of 1- and 2-month Chlorella supplementation(8 g Chlorella/day/person, equivalent to $22.9 \mathrm{mg}$ lutein/day/person).

\section{EXPERIMENTAL PROCEDURES}

\subsection{Materials}

Zeaxanthin, $\beta$-cryptoxanthin, lycopene, and lutein were purchased from Extrasynthese (Lyon, France). $\alpha$-carotene, $\beta$-carotene, echinenone, pyrogallol, methanol, ammonium acetate, and methyl tert-butyl ether (MTBE) were obtained from Wako (Osaka, Japan). Chlorella tablets (product name: Sun Chlorella $A^{\circledR}$ ) were provided by Sun Chlorella Co., Ltd. (Kyoto, Japan). Chlorella tablets, consisting of Sun Chlorella $A^{\circledR}$, were provided by Sun Chlorella Co., Ltd. (Kyoto, Japan). The daily intake of $8 \mathrm{~g}$ of tablets contained $22.9 \mathrm{mg}$ lutein and $5 \mathrm{mg} \beta$-carotene for carotenoids. This dosage was determined according to our previous studies $^{11)}$. All other reagents were of analytical grade.

\subsection{Methods}

\subsubsection{Supplementation study}

The present study was conducted according to the guidelines laid down in the Declaration of Helsinki and all procedures involving human subjects were approved by the ethics committee of the New Drug Development Research Center, Inc. (number PMS-2012-001). Exclusion criteria included pregnancy, lactation, and severe medical illness. Twelve healthy subjects (aged 50 - 68 years; 7 men and 5 women) participated in this study. Written informed consent was obtained from all subjects according to the criteria of the ethics committee.

For the Chlorella-supplemented group ( 3 men and 3 women), subjects took 40 Chlorella tablets ( $8 \mathrm{~g}$, containing $22.9 \mathrm{mg}$ lutein and $5 \mathrm{mg} \beta$-carotene) per day for a total of 2 months. For the placebo group ( 4 men and 2 women), placebo tablets ( $85 \%$ lactose) were ingested for a total of 2 months. At 1 or 2 months after ingestion of the tablets, blood was collected into a tube with heparin as an anticoagulant. The blood samples were subjected to centrifugation at $1000 \mathrm{~g}$ for $10 \mathrm{~min}$ at $4^{\circ} \mathrm{C}$. After the plasma and buffy coat were removed, erythrocytes were washed 3 times with phosphate buffered saline ( $\mathrm{pH}$ 7.4) to prepare packed cells. The packed cells were immediately subjected to determi- nation of carotenoids and PLOOH (combined values obtained for phosphatidylcholine hydroperoxide ( $\mathrm{PCOOH})$ and phosphatidylethanolamine hydroperoxide $(\mathrm{PEOOH})$ ). Plasma samples were stored at $-80^{\circ} \mathrm{C}$ for later analysis.

2.2.2 Extraction and determination of PLOOH

For the determination of erythrocyte PLOOH concentrations, total lipids were extracted from packed cells with a mixture of 2-propanol and chloroform (1:1, v/v, containing $5 \mathrm{nmol} / \mathrm{L}$ EDTA-2Na). Using total erythrocyte lipids, $\mathrm{PLOOH}(\mathrm{PCOOH}$ and $\mathrm{PEOOH}$ ) was determined by HPLC coupled with chemiluminescence (CL) detection ${ }^{4,16)}$. The column was a $4.6 \times 250 \mathrm{~mm}, 5 \mu \mathrm{m}$ Finepak SIL NH2-5 column (Japan Spectroscopic Co., Tokyo, Japan). The eluent was 2-propanol:methanol:water (135:45:20, v/v/v), and the flow rate was $1 \mathrm{~mL} / \mathrm{min}$. Post-column CL detection was carried out using a CLD-100 detector(Tohoku Electronic Industries Co., Sendai, Japan). A mixture of luminol and cytochrome $\mathrm{C}$ in $50 \mathrm{mmol} / \mathrm{L}$ borate buffer $(\mathrm{pH} 10)$ was used as a hydroperoxide-specific post-column CL reagent and the flow rate was $1.8 \mathrm{~mL} / \mathrm{min}^{16)}$. Calibration was carried out using $\mathrm{PCOOH}$ and $\mathrm{PEOOH}$ standards that were prepared by the methoxypropene method ${ }^{17)}$. Plasma $\mathrm{PCOOH}$ was measured by HPLC-CL ${ }^{18)}$.

2.2.3 Extraction and determination of carotenoids

Two $\mathrm{mL}$ of packed cells were diluted with $2 \mathrm{~mL}$ of water and were mixed with $4 \mathrm{~mL}$ of $80 \mathrm{mmol} / \mathrm{L}$ ethanolic pyrogallol, $0.8 \mathrm{~mL}$ of $1.8 \mathrm{~mol} / \mathrm{L}$ aqueous $\mathrm{KOH}$, and $125 \mu \mathrm{L}$ of one $\mu \mathrm{mol} / \mathrm{L}$ ethanolic echinenone (an internal standard). After addition of $1 \mathrm{~mL}$ of $0.1 \mathrm{~mol} / \mathrm{L}$ aqueous sodium dodecyl sulfate, the sample was mixed with $12 \mathrm{~mL}$ of hexane/dichloromethane (5:1, v/v, containing $2.4 \mathrm{mmol} / \mathrm{L}$ butylated hydroxytoluene) for extraction of erythrocyte carotenoids. The extract was purified with a Sep-Pak silica cartridge (Waters, Milford, MA), and then subjected to HPLC coupled with UV detection ${ }^{8,19,20)}$. These extraction procedures were conducted under subdued (yellow) light to minimize photo degradation of the carotenoids.

A C30 carotenoid column $(4.6 \times 250 \mathrm{~mm}, 5 \mu \mathrm{m}$; YMC, Kyoto, Japan) was used; the column was eluted using a binary gradient consisting of the following HPLC solvents: A, methanol/MTBE/water (83:15:2 v/v/v, containing 3.9 mmol/L ammonium acetate), and B, methanol/MTBE/ water (8:90:2 v/v/v, containing $2.6 \mathrm{mmol} / \mathrm{L}$ ammonium acetate $)^{19,20)}$. The gradient profile was as follows: 0-12 min, 10-55\% B linear; 12-20 min, 55-100\% B linear; 20-25 min, $100 \%$ B; $25-27$ min, 100-10\%. The flow rate was adjusted to one $\mathrm{mL} / \mathrm{min}$, and the column temperature was maintained at $30^{\circ} \mathrm{C}$. The column eluate was sent to a UV detector(UV-2075 PLUS, Japan Spectroscopic Co., Tokyo, Japan)for monitoring lutein and other carotenoids at 463 $\mathrm{nm}$. Concentrations of erythrocyte carotenoids were calculated using an equation corresponding to the standard curve of each carotenoid and were adjusted by the percentage recovery of the added echinenone (the internal 


\section{Effect of Chlorella ingestion on erythrocyte lipid oxidation}

standard). Plasma lutein and other carotenoids were determined by HPLC coupled with ultraviolet (UV) detection $^{21)}$. Plasma and erythrocyte tocopherols were measured by HPLC with fluorescence detection ${ }^{8,22)}$. Blood characteristics (parameters as given in Table 1 and Table 2) were analyzed using standardized methods.

2.2.4 Statistical analyses

Data were expressed as mean values and standard deviations. All data were analyzed by a normality test and a Friedman test followed by Scheffe's method with non-normally distributed data. Comparisons of data collected before and after supplementation were analyzed by Student's t-test with normally distributed data, and MannWhitney U test for non-normally distributed data. These statistical analyses were conducted using Excel Toukei 2010 (Social Survey Research Information, Tokyo, Japan).

\section{RESULTS}

Table 1 shows physical and haematological parameters such as blood pressure, platelet, leucocyte, neutrophil, monocyte, erythrocyte, and lymphocyte counts before and after a total of 2 months oral intake of Chlorella or placebo tablets. No change was observed after the intake of the Chlorella or placebo tablets. Table 2 shows blood biochemical parameters such as glutamate oxaloacetate transaminase (GOT), glutamate pyruvate transaminase (GPT), lactate dehydrogenase (LDH), alkaline phosphatase (ALP), guanosine 5' -triphosphate ( $\gamma$-GTP) after the intake of Chlorella or placebo tablets. No differences were observed among them, and the values were found to be within normal ranges.

As shown in Table 3, erythrocyte PLOOH concentrations $(5.0 \pm 1.7 \mathrm{pmol} / \mathrm{mL}$ packed cells) were significantly lower after a 2 months oral intake of $8 \mathrm{~g}$ Chlorella/day than before the supplementation $(11.9 \pm 5.5 \mathrm{pmol} / \mathrm{mL}$ packed cells). No difference was observed in erythrocyte PLOOH concentrations after 1 month of Chlorella intake (7.9 \pm 3.7 $\mathrm{pmol} / \mathrm{mL}$ packed cells). In the erythrocytes of the placebo group, no change in PLOOH concentrations was observed after treatment. For erythrocyte carotenoids, 6 carotenoids (lutein, zeaxanthin, $\beta$-cryptoxanthin, $\alpha$-carotene, $\beta$-carotene, and lycopene) were detected (Table 3). Among these carotenoids, lutein was the predominant xanthophyll found, and it increased 4.6-fold (from 49.7 to $230.7 \mathrm{pmol} /$ $\mathrm{mL}$ packed cells) after a total of 2 months of Chlorella ingestion. Erythrocyte tocopherol levels were not changed after Chlorella ingestion. These results indicate that daily Chlorella intake would be effective for improving and maintaining erythrocyte antioxidant status and lutein concentrations in humans.

Table 4 shows plasma $\mathrm{PCOOH}$, carotenoids and tocopherol concentrations before and after a total of 2 months of
Chlorella ingestion. PCOOH is a representative lipid hydroperoxide present in plasma; concentrations were not changed after Chlorella ingestion or placebo treatment. For plasma carotenoids, concentrations of lutein, $\alpha$-carotene, and $\beta$-carotene significantly increased (4.4-fold for lutein, 2.1-fold for $\alpha$-carotene, 1.8 -fold for $\beta$-carotene) after Chlorella supplementation(Table 4). In the placebo group, no changes were observed for plasma carotenoids or tocopherols after the treatment (Table 4).

\section{DISCUSSION}

In the present study, we conducted a randomized, double-blind, placebo-controlled human trial to investigate the impact of 1 and 2 months of Chlorella supplementation on carotenoid compositions and PLOOH concentrations in erythrocytes as well as the plasma of 12 senior healthy subjects. Subjects ingested 8 g Chlorella/day/person, equivalent to $22.9 \mathrm{mg}$ lutein/day/person. This research was carried out to assess the possibility that Chlorella ingestion could prevent the increase of erythrocyte PLOOH in senior Japanese volunteers.

Previously, we discovered that erythrocyte PLOOHs in $\mathrm{AD}$ patients were significantly higher than in normal subjects $^{4,9)}$. In normal volunteers, erythrocyte PLOOH of senior subjects (56-92 of age) was higher than the young subjects $(21-27 \text { of age })^{16)}$. The magnitude of PLOOH change is not proportional to subject body weight. $\mathrm{PCOOH}$ and $\mathrm{PEOOH}$ are the major PLOOHs in human erythrocytes, and $\mathrm{PCOOH}$ is the principal $\mathrm{PLOOH}$ in human plasma ${ }^{4,9)}$. The data suggested that biochemically older erythrocytes circulated in the blood of patients with senile dementia than in normal subjects. Lutein is a polar carotenoid and important antioxidant present in erythrocyte lipid bilay$\mathrm{ers}^{8)}$. We analyzed the effect of Chlorella supplementation as a lutein-rich nutrient (contains an average of $0.29 \%$ by dry weight) on the prevention of erythrocyte membrane lipid oxidation.

In the present study, Chlorella intake resulted in no meaningful changes of blood biochemistry or parameters (Table 1 and Table 2). These data might indicate the safety of Chlorella supplementation. As shown in Table 3, Chlorella supplementation was effective at elevating the lutein concentration in the erythrocyte lipid bilayers. The increased erythrocyte $\beta$-cryptoxanthin concentrations found after ingestion might have been due to food intake (such as mandarin oranges, rich in $\beta$-cryptoxanthin) by two volunteers during the ingestion period (from October $15^{\text {th }}$ to December $\left.17^{\text {th }}\right)$. Decreased lycopene concentrations found in erythrocytes and plasma after the 2-month Chlorella treatment were recognized due to the lack of lycopene in the ingested Chlorella tablets and meals during the study (Table 3 and Table 4). Erythrocyte and plasma 
Table 1 Physical and haematological paramaters before and after a total of 2 months oral intake of 0 (placebo) or $8 \mathrm{~g}$ /day Chlorella (Mean values and standard deviations).

\begin{tabular}{|c|c|c|c|c|c|c|}
\hline \multicolumn{2}{|c|}{ Daily chlorella supplementation } & \multicolumn{2}{|c|}{$0 \mathrm{~g}$ (placebo group) } & \multicolumn{2}{|c|}{$8 \mathrm{~g}$} & \multirow[b]{2}{*}{$p \ddagger$} \\
\hline \multicolumn{2}{|l|}{ Parameters } & Mean & SD & Mean & SD & \\
\hline \multicolumn{2}{|l|}{ Age (years) } & 57 & 7 & 58 & 7 & 0.840 \\
\hline \multicolumn{2}{|l|}{ Total number of subjects } & 6 & & 6 & & \\
\hline \multicolumn{2}{|l|}{ Men } & 4 & & 3 & & \\
\hline \multicolumn{2}{|l|}{ Women } & 2 & & 3 & & \\
\hline \multicolumn{2}{|l|}{ Height $(\mathrm{cm})$} & 161.9 & 10.0 & 161.8 & 6.8 & 0.984 \\
\hline \multirow[t]{3}{*}{ Weight (kg) } & 0 month & 59.3 & 10.4 & 61.4 & 11.5 & 0.747 \\
\hline & 1 & 60.0 & 12.1 & 61.8 & 11.2 & 0.791 \\
\hline & 2 & 60.1 & 12.6 & 61.7 & 11.2 & 0.819 \\
\hline \multirow[t]{3}{*}{ BMI $\left(\mathrm{kg} / \mathrm{m}^{2}\right)$} & 0 month & 22.5 & 2.5 & 23.4 & 3.4 & 0.728 \\
\hline & 1 & 22.7 & 2.7 & 23.5 & 3.2 & 0.654 \\
\hline & 2 & 22.7 & 2.7 & 23.5 & 3.2 & 0.681 \\
\hline \multirow[t]{3}{*}{ Systolic blood pressure (mmHg) } & 0 month & 112.2 & 8.9 & 130.2 & 18.6 & 0.069 \\
\hline & 1 & 116.5 & 12.3 & 127.8 & 24.1 & 0.338 \\
\hline & 2 & 110.5 & 13.9 & 128.5 & 31.3 & 0.239 \\
\hline \multirow[t]{3}{*}{ Diastolic blood pressure $(\mathrm{mmHg})$} & 0 month & 74.2 & 9.0 & 83.3 & 14.5 & 0.224 \\
\hline & 1 & 75.7 & 9.8 & 81.5 & 12.0 & 0.380 \\
\hline & 2 & 72.5 & 11.2 & 82.2 & 18.4 & 0.304 \\
\hline \multirow{3}{*}{ Platelets $\left(\times 10^{4} / \mu \mathrm{L}\right)$} & 0 month & 22.9 & 5.4 & 23.6 & 6.8 & 0.851 \\
\hline & 1 & 24.1 & 5.2 & 24.9 & 5.9 & 0.801 \\
\hline & 2 & 24.8 & 5.3 & 24.4 & 6.7 & 0.900 \\
\hline Leucocytes $(/ \mu \mathrm{L})$ & 0 month & 4716.7 & 762.7 & 4483.3 & 643.2 & 0.580 \\
\hline & 1 & 4500.0 & 784.9 & 5483.3 & 980.6 & 0.086 \\
\hline & 2 & 4400.0 & 469.0 & 4650.0 & 653.5 & 0.466 \\
\hline Neutrophil (\%) & 0 month & 64.0 & 9.8 & 52.2 & 10.0 & 0.065 \\
\hline & 1 & 60.7 & 7.6 & 60.1 & 6.9 & 0.883 \\
\hline & 2 & 62.6 & 7.4 & 52.9 & 8.1 & 0.056 \\
\hline Eosinophil granulocyte (\%) & 0 month & 2.7 & 2.1 & 2.5 & 0.9 & 0.863 \\
\hline & 1 & 2.5 & 1.5 & 2.8 & 1.1 & 0.676 \\
\hline & 2 & 2.5 & 1.4 & 3.9 & 2.5 & 0.275 \\
\hline Basophil granulocyte (\%) & 0 month & 0.5 & 0.4 & 0.6 & 0.4 & 0.567 \\
\hline & 1 & 0.5 & 0.3 & 0.6 & 0.3 & 0.783 \\
\hline & 2 & 0.6 & 0.3 & 0.8 & 0.4 & 0.472 \\
\hline Monocyte (\%) & 0 month & 6.0 & 1.7 & 5.9 & 1.0 & 0.843 \\
\hline & 1 & 5.7 & 1.4 & 4.7 & 1.1 & 0.208 \\
\hline & 2 & 5.6 & 1.4 & 5.7 & 1.0 & 0.910 \\
\hline Erythrocytes $\left(\times 10^{4} / \mu \mathrm{L}\right)$ & 0 month & 466.0 & 27.0 & 450.8 & 35.8 & 0.428 \\
\hline & 1 & 466.8 & 21.1 & 455.8 & 32.6 & 0.506 \\
\hline & 2 & 459.0 & 30.3 & 451.8 & 46.7 & 0.760 \\
\hline lymphocyte & 0 month & 26.8 & 9.6 & 38.8 & 9.6 & 0.057 \\
\hline & 1 & 30.6 & 7.1 & 31.9 & 6.9 & 0.760 \\
\hline & 2 & 28.8 & 6.4 & 36.8 & 7.4 & 0.073 \\
\hline $\mathrm{Hb}(\mathrm{g} / \mathrm{dL})$ & 0 month & 14.0 & 1.3 & 13.8 & 1.0 & 0.756 \\
\hline & 1 & 14.2 & 1.2 & 14.1 & 1.1 & 0.941 \\
\hline & 2 & 13.9 & 1.5 & 13.8 & 1.5 & 0.882 \\
\hline Haematocrit (\%) & 0 month & 42.7 & 2.9 & 42.0 & 2.8 & 0.680 \\
\hline & 1 & 42.9 & 2.2 & 42.4 & 3.0 & 0.743 \\
\hline & 2 & 42.3 & 3.6 & 42.3 & 4.2 & 1.000 \\
\hline Total bilirubin (mg/dL) & 0 month & 0.7 & 0.1 & 0.7 & 0.3 & 0.900 \\
\hline & 1 & 0.7 & 0.2 & 0.7 & 0.2 & 1.000 \\
\hline & 2 & 0.6 & 0.1 & 0.8 & 0.3 & 0.245 \\
\hline MCV (fL) & 0 month & 91.7 & 4.6 & 93.4 & 4.4 & 0.535 \\
\hline & 1 & 92.1 & 4.4 & 93.1 & 4.6 & 0.690 \\
\hline & 2 & 92.2 & 5.1 & 93.7 & 3.7 & 0.566 \\
\hline $\mathrm{MCH}(\mathrm{pg})$ & 0 month & 30.0 & 2.2 & 30.6 & 1.4 & 0.599 \\
\hline & 1 & 30.4 & 2.3 & 31.0 & 1.8 & 0.451 \\
\hline & 2 & 32.0 & 2.5 & 30.6 & 1.5 & 0.274 \\
\hline $\mathrm{MCHC}(\%)$ & 0 month & 32.7 & 1.0 & 32.7 & 1.0 & 0.955 \\
\hline & 1 & 33.0 & 1.2 & 33.3 & 0.9 & 0.588 \\
\hline & 2 & 32.9 & 1.1 & 32.6 & 0.9 & 0.630 \\
\hline
\end{tabular}

* Student's t-test among groups.

$\mathrm{BMI}$, body mass index; $\mathrm{Hb}$, hemoglobin; $\mathrm{MCV}$, mean corpuscular volume; $\mathrm{MCH}$, mean corpuscular hemoglobin; $\mathrm{MCHC}$, mean corpuscular hemoglobin concentration. 
Table 2 Blood biochemical paramaters before and after a total of 2 months oral intake in the 0 (placebo) or $8 \mathrm{~g}$ /day Chlorella (Mean values and standard deviations).

\begin{tabular}{|c|c|c|c|c|c|c|}
\hline \multicolumn{2}{|c|}{ Daily chlorella supplementation } & \multicolumn{2}{|c|}{$0 \mathrm{~g}$ (placebo group) } & \multicolumn{2}{|c|}{$8 \mathrm{~g}$} & \multirow[b]{2}{*}{$p \ddagger$} \\
\hline \multicolumn{2}{|c|}{ Parameters } & Mean & $\mathrm{SD}$ & Mean & SD & \\
\hline \multirow[t]{3}{*}{ Total protein $(\mathrm{g} / \mathrm{dL})$} & 0 month & 7.3 & 0.3 & 7.4 & 0.3 & 0.474 \\
\hline & 1 & 7.4 & 0.3 & 7.5 & 0.2 & 0.645 \\
\hline & 2 & 7.3 & 0.5 & 7.3 & 0.3 & 0.950 \\
\hline \multirow[t]{3}{*}{ Albumin $(\mathrm{g} / \mathrm{dL})$} & 0 month & 4.4 & 0.2 & 4.4 & 0.3 & 0.769 \\
\hline & 1 & 4.5 & 0.3 & 4.4 & 0.3 & 0.510 \\
\hline & 2 & 4.4 & 0.4 & 4.3 & 0.4 & 0.785 \\
\hline \multirow[t]{3}{*}{ Creatinine $(\mathrm{mg} / \mathrm{dL})$} & 0 month & 0.7 & 0.1 & 0.7 & 0.2 & 0.973 \\
\hline & 1 & 0.8 & 0.1 & 0.7 & 0.2 & 0.785 \\
\hline & 2 & 0.8 & 0.1 & 0.8 & 0.2 & 0.973 \\
\hline \multirow[t]{3}{*}{ Homocysteine $(\mathrm{nmol} / \mathrm{mL})$} & 0 month & 13.8 & 1.1 & 15.0 & 8.0 & 0.728 \\
\hline & 1 & 13.3 & 1.0 & 15.0 & 9.7 & 0.683 \\
\hline & 2 & 13.0 & 1.4 & 14.8 & 7.5 & 0.579 \\
\hline \multirow[t]{3}{*}{ GOT (U/L) } & 0 month & 24.5 & 10.5 & 20.8 & 3.5 & 0.447 \\
\hline & 1 & 22.5 & 5.5 & 21.7 & 3.7 & 0.765 \\
\hline & 2 & 23.7 & 3.5 & 20.5 & 1.5 & 0.083 \\
\hline \multirow[t]{3}{*}{ GPT (U/L) } & 0 month & 19.8 & 9.7 & 22.0 & 8.9 & 0.696 \\
\hline & 1 & 20.7 & 7.1 & 22.0 & 7.6 & 0.761 \\
\hline & 2 & 22.7 & 4.8 & 21.7 & 6.2 & 0.761 \\
\hline \multirow[t]{3}{*}{ LDH (U/L) } & 0 month & 198.2 & 35.9 & 191.8 & 22.6 & 0.723 \\
\hline & 1 & 187.8 & 23.5 & 188.3 & 29.8 & 0.975 \\
\hline & 2 & 189.7 & 21.5 & 185.3 & 22.0 & 0.737 \\
\hline \multirow[t]{3}{*}{ ALP (U/L) } & 0 month & 181.3 & 21.0 & 235.5 & 34.4 & 0.011 \\
\hline & 1 & 183.0 & 31.3 & 254.3 & 47.2 & 0.014 \\
\hline & 2 & 184.5 & 39.9 & 245.7 & 50.5 & 0.044 \\
\hline \multirow[t]{3}{*}{$\gamma$-GTP (U/L) } & 0 month & 26.2 & 12.7 & 32.7 & 14.7 & 0.433 \\
\hline & 1 & 27.0 & 9.1 & 34.2 & 18.1 & 0.414 \\
\hline & 2 & 27.2 & 11.0 & 32.3 & 19.7 & 0.591 \\
\hline \multirow[t]{3}{*}{ CPK (U/L) } & 0 month & 208.2 & 234.1 & 108.2 & 42.6 & 0.348 \\
\hline & 1 & 139.0 & 56.7 & 104.0 & 63.5 & 0.338 \\
\hline & 2 & 148.3 & 75.7 & 90.0 & 27.6 & 0.124 \\
\hline \multirow[t]{3}{*}{ Uric acid (mg/dL) } & 0 month & 5.1 & 1.0 & 5.2 & 2.2 & 0.922 \\
\hline & 1 & 5.4 & 0.7 & 5.1 & 1.9 & 0.743 \\
\hline & 2 & 5.7 & 1.2 & 5.1 & 1.5 & 0.460 \\
\hline \multirow[t]{3}{*}{ Urea (mg/dL) } & 0 month & 13.6 & 2.7 & 14.1 & 4.1 & 0.804 \\
\hline & 1 & 13.6 & 1.7 & 12.4 & 1.6 & 0.209 \\
\hline & 2 & 14.4 & 2.6 & 13.6 & 1.6 & 0.510 \\
\hline \multirow[t]{3}{*}{ Glucose (mg/dL) } & 0 month & 82.8 & 7.7 & 89.3 & 9.8 & 0.231 \\
\hline & 1 & 83.5 & 6.1 & 89.3 & 9.4 & 0.236 \\
\hline & 2 & 85.3 & 9.6 & 89.2 & 10.9 & 0.534 \\
\hline \multirow[t]{3}{*}{ Total cholesterol (mg/dL) } & 0 month & 226.0 & 7.7 & 219.0 & 31.3 & 0.615 \\
\hline & 1 & 228.8 & 11.7 & 219.3 & 29.8 & 0.493 \\
\hline & 2 & 225.5 & 9.9 & 216.3 & 30.5 & 0.510 \\
\hline HDL cholesterol (mg/dL) & 0 month & 73.0 & 19.4 & 58.5 & 14.5 & 0.176 \\
\hline & 1 & 75.3 & 17.0 & 56.0 & 13.1 & 0.053 \\
\hline & 2 & 73.2 & 16.7 & 57.7 & 14.7 & 0.119 \\
\hline LDL cholesterol (mg/dL) & 0 month & 134.7 & 20.6 & 135.7 & 27.9 & 0.945 \\
\hline & 1 & 136.7 & 24.1 & 136.7 & 28.0 & 1.000 \\
\hline & 2 & 136.0 & 23.1 & 130.8 & 25.0 & 0.718 \\
\hline TG (mg/dL) & 0 month & 77.2 & 35.2 & 124.7 & 78.8 & 0.220 \\
\hline & 1 & 66.5 & 13.0 & 156.0 & 75.8 & 0.034 \\
\hline & 2 & 62.2 & 18.7 & 145.7 & 94.6 & 0.084 \\
\hline
\end{tabular}

Student's t-test among groups.

CPK, creatine phosphokinase; TG, triglyceride. 
Table 3 Erythrocyte phospholipid hydroperoxides (PLOOH), carotenoids and tocopherol levels in the subjects before and after a total of 2 months oral intake of the 0 (placebo) or $8 \mathrm{~g} /$ day Chlorella (Mean values and standard deviations).

\begin{tabular}{|c|c|c|c|c|c|c|}
\hline \multicolumn{2}{|c|}{ Daily chlorella supplementation } & \multicolumn{2}{|c|}{$0 \mathrm{~g}$ (placebo group) } & \multicolumn{2}{|c|}{$8 \mathrm{~g}$} & \multirow[b]{2}{*}{$p \dagger$} \\
\hline \multicolumn{2}{|l|}{ Parameters } & Mean & SD & Mean & SD & \\
\hline \multicolumn{7}{|l|}{ PLOOH (pmol/mL packed cells) } \\
\hline \multirow[t]{3}{*}{$\mathrm{PCOOH}$} & 0 month & 8.2 & 4.1 & 8.4 & 4.2 & 0.936 \\
\hline & 1 & 9.7 & 8.8 & 6.6 & 3.2 & 0.749 \\
\hline & 2 & 4.1 & 2.2 & 3.7 & 1.3 & 0.936 \\
\hline \multirow[t]{3}{*}{$\mathrm{PEOOH}$} & 0 month & 1.1 & 0.4 & 3.5 & 3.8 & 0.261 \\
\hline & 1 & 1.4 & 0.8 & 1.2 & 0.7 & 0.630 \\
\hline & 2 & 1.0 & 0.9 & 1.3 & 1.0 & 0.336 \\
\hline \multirow[t]{3}{*}{$\mathrm{PLOOH}(\mathrm{PCOOH}+\mathrm{PEOOH})$} & 0 month & 9.3 & 4.3 & 11.9 & 5.5 & 0.423 \\
\hline & 1 & 11.1 & 9.0 & 7.9 & 3.7 & 0.747 \\
\hline & 2 & 5.1 & 2.9 & $5.0^{*}$ & 1.7 & 0.522 \\
\hline \multicolumn{7}{|c|}{ Carotenoids (pmol/mL packed cells) } \\
\hline \multirow[t]{3}{*}{ Lutein } & 0 month & 47.4 & 13.2 & 49.7 & 29.9 & 0.873 \\
\hline & 1 & 54.3 & 19.8 & $192.9^{*}$ & 122.8 & 0.109 \\
\hline & 2 & 55.5 & 26.3 & 230.7 & 129.4 & 0.025 \\
\hline \multirow[t]{3}{*}{ Zeaxanthin } & 0 month & 9.4 & 5.2 & 9.2 & 3.5 & 1.000 \\
\hline & 1 & 8.6 & 3.0 & 11.1 & 7.7 & 0.631 \\
\hline & 2 & 11.2 & 6.5 & 7.1 & 3.1 & 0.200 \\
\hline \multirow[t]{3}{*}{$\beta$-Cryptoxanthin } & 0 month & 3.9 & 2.1 & 2.0 & 0.8 & 0.078 \\
\hline & 1 & 4.2 & 2.9 & 3.5 & 1.8 & 0.522 \\
\hline & 2 & 6.5 & 4.5 & $7.3^{*}$ & 3.9 & 0.749 \\
\hline \multirow[t]{3}{*}{$\alpha$-Carotene } & 0 month & 0.7 & 0.7 & 1.8 & 2.2 & 0.262 \\
\hline & 1 & 0.8 & 0.4 & 1.6 & 1.4 & 0.337 \\
\hline & 2 & 1.0 & 0.9 & 3.2 & 2.5 & 0.150 \\
\hline \multirow[t]{3}{*}{$\beta$-Carotene } & 0 month & 4.2 & 3.1 & 4.7 & 3.0 & 1.000 \\
\hline & 1 & 4.4 & 2.4 & 9.8 & 13.1 & 0.631 \\
\hline & 2 & 5.9 & 5.6 & 6.9 & 4.2 & 0.522 \\
\hline \multirow[t]{3}{*}{ Lycopene } & 0 month & 4.6 & 0.5 & 4.7 & 0.7 & 0.522 \\
\hline & 1 & 4.8 & 1.2 & 4.9 & 1.3 & 0.749 \\
\hline & 2 & 4.8 & 0.7 & $3.7^{*}$ & 0.3 & 0.004 \\
\hline \multicolumn{7}{|c|}{ Tocopherols (nmol/mL packed cells) } \\
\hline \multirow[t]{3}{*}{$\alpha$-Tocopherol } & 0 month & 4.2 & 1.7 & 5.4 & 1.2 & 0.262 \\
\hline & 1 & 4.5 & 1.7 & 7.6 & 1.2 & 0.010 \\
\hline & 2 & 5.8 & 1.7 & 6.9 & 1.9 & 0.337 \\
\hline \multirow[t]{3}{*}{$\gamma$-Tocopherol } & 0 month & 0.9 & 0.4 & 1.2 & 0.5 & 0.423 \\
\hline & 1 & 0.9 & 0.7 & 1.7 & 0.7 & 0.037 \\
\hline & 2 & 1.6 & 0.8 & $1.6^{\S}$ & 0.6 & 1.000 \\
\hline
\end{tabular}

Mean values were significantly different in the Friedman test with Scheffe's method between before administration and 1 or 2 month after administration. $* p<0.05$

Mean values were significantly different in the Friedman test with Scheffe's method between 1 month administration and 2 month after administration. $\S p<0.05$

$\dagger$ Mann-Whitney U test among groups.

tocopherol concentrations were recognized to reflect the contents of daily meals during the experimental period (Table 3 and Table 4). This may reflect the scarcity of any tocopherols in the Chlorella tablets employed in the present study.

After the 2-month treatment, erythrocyte PLOOH con- centrations in the Chlorella group were significantly reduced $(5.0 \pm 1.7 \mathrm{pmol} / \mathrm{mL}$ packed cells vs. $11.9 \pm 5.5$ $\mathrm{pmol} / \mathrm{mL}$ packed cells). The Chlorella group initially showed rather high values compared to the placebo group (9.3 $\pm 4.3 \mathrm{pmol} / \mathrm{mL}$ packed cells). Comparing the placebo and Chlorella groups after 2 months of treatment, there 
Table 4 Plasma phospholipid hydroperoxides (PLOOH), carotenoids and tocopherol levels in the subjects before and after a total of 2 months oral intake of 0 (placebo) or $8 \mathrm{~g}$ /day Chlorella (Mean values and standard deviations).

\begin{tabular}{|c|c|c|c|c|c|c|}
\hline \multicolumn{2}{|c|}{ Daily chlorella supplementation } & \multicolumn{2}{|c|}{$0 \mathrm{~g}$ (placebo group) } & \multicolumn{2}{|c|}{$8 \mathrm{~g}$} & \multirow[b]{2}{*}{$p \dagger$} \\
\hline \multicolumn{2}{|c|}{ Parameters } & Mean & $\mathrm{SD}$ & Mean & SD & \\
\hline \multicolumn{7}{|c|}{ PLOOH (pmol/mL plasma) } \\
\hline \multicolumn{7}{|l|}{$\mathrm{PCOOH}$} \\
\hline & 0 month & 171.1 & 59.8 & 115.1 & 23.6 & 0.150 \\
\hline & 1 & 138.1 & 12.0 & 115.6 & 14.5 & 0.025 \\
\hline & 2 & 133.3 & 33.6 & 143.1 & 55.5 & 0.631 \\
\hline \multicolumn{7}{|c|}{ Carotenoids (pmol/mL plasma) } \\
\hline \multirow[t]{3}{*}{ Lutein } & 0 month & 351.3 & 73.8 & 370.1 & 199.6 & 0.749 \\
\hline & 1 & 408.2 & 137.5 & $1523.9^{*}$ & 648.8 & 0.055 \\
\hline & 2 & 430.5 & 213.3 & $1620.3^{*}$ & 926.3 & 0.037 \\
\hline \multirow[t]{3}{*}{ Zeaxanthin } & 0 month & 84.7 & 61.6 & 127.1 & 29.6 & 0.200 \\
\hline & 1 & 69.3 & 28.6 & 78.8 & 56.3 & 1.000 \\
\hline & 2 & 88.4 & 47.2 & 81.4 & 52.7 & 0.749 \\
\hline \multirow[t]{3}{*}{$\beta$-Cryptoxanthin } & 0 month & 111.1 & 67.6 & 106.7 & 68.2 & 0.631 \\
\hline & 1 & 172.4 & 127.7 & 151.4 & 54.0 & 0.873 \\
\hline & 2 & 209.4 & 109.2 & 298.2 & 197.6 & 0.522 \\
\hline \multirow[t]{3}{*}{$\alpha$-Carotene } & 0 month & 130.5 & 56.1 & 180.3 & 173.3 & 1.000 \\
\hline & 1 & 109.1 & 46.8 & $364.3^{*}$ & 184.7 & 0.025 \\
\hline & 2 & 99.5 & 39.0 & $382.5^{*}$ & 231.3 & 0.037 \\
\hline \multirow[t]{3}{*}{$\beta$-Carotene } & 0 month & 380.1 & 118.3 & 371.7 & 220.1 & 0.749 \\
\hline & 1 & 411.2 & 229.9 & $644.2^{*}$ & 326.6 & 0.262 \\
\hline & 2 & 414.9 & 270.2 & $659.6^{*}$ & 358.9 & 0.150 \\
\hline \multirow[t]{3}{*}{ Lycopene } & 0 month & 250.0 & 136.3 & 226.7 & 112.3 & 0.873 \\
\hline & 1 & 259.5 & 89.7 & 154.2 & 78.6 & 0.078 \\
\hline & 2 & 199.2 & 77.7 & $106.3^{*}$ & 52.3 & 0.037 \\
\hline \multicolumn{7}{|c|}{ Tocopherols (nmol/mL plasma) } \\
\hline \multirow[t]{3}{*}{$\alpha$-Tocopherol } & 0 month & 24.6 & 5.4 & 29.0 & 7.0 & 0.423 \\
\hline & 1 & 22.1 & 4.6 & 29.2 & 8.3 & 0.078 \\
\hline & 2 & 26.1 & 3.6 & 28.7 & 7.1 & 1.000 \\
\hline \multirow[t]{3}{*}{$\gamma$-Tocopherol } & 0 month & 6.0 & 2.2 & 7.4 & 4.3 & 0.749 \\
\hline & 1 & 4.4 & 2.6 & 5.0 & 1.9 & 0.631 \\
\hline & 2 & 6.3 & 2.3 & 5.7 & 1.3 & 0.423 \\
\hline
\end{tabular}

Mean values were significantly different in the Friedman test with Scheffe's method between before administration and 1 or 2 month after administration. ${ }^{*} p<0.05$

$\dagger$ Mann-Whitney U test among groups.

was no difference in PLOOH concentrations. However, we confirmed a significant decrease of PLOOH after the 2 months of Chlorella ingestion(Table 3). There was a wide dispersion of $\mathrm{PLOOH}$ data found in the placebo group (Table 3).

Our results indicate for the first time that Chlorella ingestion improves erythrocyte antioxidant status and lowers the PLOOH concentration. Thus, Chlorella supplementation could maintain the normal function of erythrocytes.
Generally, aged erythrocytes contain more oxidized lipids than young erythrocytes ${ }^{16)}$. Chlorella intake may prevent the development of senile dementia, including $\mathrm{AD}$, due to its suppression of the accelerated aging of erythrocytes in senior subjects. 


\section{T. Miyazawa, K. Nakagawa, H. Takekoshi et al.}

\section{CONCLUSIONS}

In summary, we performed a randomized, double-blind, placebo-controlled human trial to investigate the impact of a total of 2 months Chlorella supplementation on erythrocytes antioxidant status. The present study provides the first evidence that Chlorella is an effective dietary source of antioxidant carotenoids that prevents PLOOH accumulation in the erythrocytes of senior Japanese subjects. We previously found that there was a higher accumulation of $\mathrm{PLOOH}$, an oxidative stress marker, in erythrocytes of dementia patients ${ }^{4,9)}$. Erythrocytes high in lipid hydroperoxides have been suggested to have a decreased ability to transport oxygen to the brain and may impair blood rheology, thus facilitating dementia. The present findings confirm the efficacy of Chlorella supplementation for the prevention of erythrocyte lipid oxidation in senile dementia, including $\mathrm{AD}$.

\section{ACKNOWLEDGMENT}

The present study was supported in part by a Grant-inAid for Scientific Research (KAKENHI: 20228002 to TM) of the Japanese Society for the Promotion of Science (JSPS, Tokyo, Japan).

\section{References}

1) Selkoe, D. J. Alzheimer's Disease: Genes, Proteins, and Therapy. Physiol. Rev. 81, 741-766 (2001).

2) Bosman, G. J.; Bartholomeus I. G.; de Man A. J.; van Kalmthout P. J.; de Grip W. J. Erythrocyte membrane characteristics indicate abnormal cellular aging in patients with Alzheimer's disease. Neurobiol. Aging 12, 13-18(1991).

3) Goodall, H. B.; Reid, A. H.; Findlay, D. J.; Hind, C.; Kay, J.; Coghill, G. Irregular distortion of the erythrocytes (acanthocytes, spur cells) in senile dementia. Dis. Markers 12, 23-41 (1994).

4) Miyazawa, T.; Suzuki, T.; Yasuda, K; Fujimoto, K.; Meguro, K.; Sasaki, H. Accumulation of phospholipid hydroperoxides in red blood cell membranes in Alzheimer disease. In Oxygen Radicals (Yagi, K.; Kondo, M.; Niki, E.; Yoshikawa, T. eds.), Elsevier Sci. Publ., Amsterdam, pp. 327-330 (1992).

5) Solerte, S. B.; Ceresini, G.; Ferrari, E.; Fioravanti, M. Hemorheological changes and overproduction of cytokines from immune cells in mild to moderate dementia of the Alzheimer's type: adverse effects on cerebromicrovascular system. Neurobiol. Aging 21, 271-281 (2000).

6) Ajmani, R. S.; Metter, E. J.; Jaykumar, R.; Ingram, D. K.; Spangler, E. L.; Abugo, O. O.; Rifkind, J. M. Hemody- namic changes during aging associated with cerebral blood flow and impaired cognitive function. Neurobiol. Aging 21, 257-269(2000).

7) Mohanty, J. G.; Eckley, D. M.; Williamson, J. D.; Launer, L.J.; Rifkind, J.M. Do red blood cell-beta-amyloid interactions alter oxygen delivery in Alzheimer' s disease? Adv. Exp. Med. Biol. 614, 29-35 (2008).

8) Nakagawa, K.; Kiko, T.; Hatade, K.; Asai, A.; Kimura, F.; Sookwong, P.; Tsuduki, T.; Arai, H.; Miyazawa, T. Development of a high-performance liquid chromatography-based assay for carotenoids in human red blood cells: application to clinical studies. Anal. Biochem. 381, 129-134 (2008).

9) Kiko, T.; Nakagawa, K.; Tsuduki, T.; Suzuki, T.; Arai, H.; Miyazawa, T. Significance of lutein in red blood cells of Alzheimer's disease patients. J. Alzheimers Dis. 28, 593-600 (2012).

10) Nakagawa, K.; Kiko, T.; Miyazawa, T.; Sookwong, P.; Tsuduki, T.; Satoh, A.; Miyazawa, T. Amyloid $\beta$-induced erythrocytic damage and its attenuation by carotenoids. FEBS Lett. 585, 1249-1254(2011).

11) Nakagawa, K.; Kiko, T.; Hatade, K.; Sookwong, P.; Arai, H.; Miyazawa, T. Antioxidant effect of lutein towards phospholipid hydroperoxidation in human erythrocytes. Brit. J. Nutr. 102, 1280-1284(2009).

12) Borowitzka, M. A.; Commercial production of microalgae: ponds, tanks, tubes and fermenters. J. Biotechnol. 70, 313-321 (1999).

13) Del Campo, J. A.; Rodríguez, H.; Moreno, J.; Vargas, M. A.; Rivas, J.; Guerrero, M. G. Accumulation of astaxanthin and lutein in Chlorella zofingiensis (Chlorophyta). Appl. Microbiol. Biotechnol . 64, 848-854(2004).

14) Bhosale, P.; Bernstein, P. S. Microbial xanthophylls. Appl. Microbiol. Biotechnol. 68, 445-455(2005).

15) Shi, X. M.; Jiang, Y.; Chen, F. High-yield production of lutein by the green microalga Chlorella protothecoides in heterotrophic fed-batch culture. Biotechnol. Prog. 18, 723-727 (2002).

16) Miyazawa T.; Suzuki T.; Fujimoto K.; Kinoshita M. Agerelated change of phosphatidylcholine hydroperoxide and phosphatidylethanolamine hydroperoxide levels in normal human red blood cells. Mech. Ageing Dev. 86, 145-150 (1996).

17) Ibusuki, D.; Nakagawa, K.; Asai, A.; Oikawa, S.; Masuda, Y.; Suzuki, T.; Miyazawa, T. Preparation of pure lipid hydroperoxides. J. Lipid Res. 49, 2668-2677 (2008).

18) Miyazawa T. Determination of phospholipid hydroperoxides in human blood plasma by a chemiluminescence-HPLC assay. Free Radic. Biol. Med. 7, 209-217 (1989).

19) Miyazawa, T.; Nakagawa, K.; Miyazawa, T. Liquid Chromatography-based Assay for Carotenoids in Human Blood. In Food and Nutritional Components in FO- 
cus No. 1. Vitamin A and Carotenoids: Chemistry, Analysis, Function and Effects.(Preedy, V. R. ed.), The Royal Society of Chemistry Publ., UK, pp. 184-203 (2012).

20) Miyazawa, T.; Nakagawa, K.; Kimura, F.; Satoh, A.; Miyazawa, T. Erythrocytes carotenoids after astaxanthin supplementation in middle-aged and senior Japanese subjects. J. Oleo Sci. 60, 495-499 (2011).

21) Miyazawa, T.; Nakagawa, K.; Kimura, F.; Satoh, A.; Mi- yazawa T. Plasma carotenoid concentrations before and after supplementation with astaxanthin in middleaged and senior subjects. Biosci. Biotechnol. Biochem. 75, 1856-1858(2011).

22) Kawakami, Y.; Tsuduki, T.; Nakagawa, K.; Miyazawa, T. Distribution of tocotrienols in rats fed a rice bran tocotrienol concentrate. Biosci. Biotechnol. Biochem. 71, 464-471 (2007). 